\title{
Clinical Etiologies, Microbial Spectrum, Antibiotic Susceptibilities, and Visual Acuity Outcomes of Acute Endophthalmitis
}

\author{
Louise J. Lu, ${ }^{1}$ Xuejing Chen, ${ }^{2,3}$ and Ron A. Adelman ${ }^{1}$
}

\begin{abstract}
Purpose: The purpose of this study was to report the clinical etiologies, microbial spectrum, antibiotic resistance, and visual acuity (VA) outcomes associated with acute endophthalmitis.

Methods: A retrospective chart review of patients with International Classification of Diseases (ICD)-9 and ICD-10 codes for endophthalmitis over a 6-year period (2011-2016) at a tertiary referral center was performed. The clinical records were reviewed to evaluate clinical etiologies, microbial spectrum, antibiotic susceptibilities and resistance, and visual outcomes.

Results: Medical records of 94 patients treated for culture-proven endophthalmitis were reviewed. The etiologies of endophthalmitis were exogenous in $68.8 \%$ of cases and endogenous in $31.2 \%$ of cases. The most common inciting factors for exogenous endophthalmitis were progression of corneal ulcer and postoperative infection after cataract extraction. The microbial spectrum of causative organisms was dominated by coagulasenegative Staphylococcus (30.9\%), followed by Staphylococcus aureus (23.4\%). The most frequent fungal isolates were Candida species. Antibiotic susceptibilities of gram-positive bacteria ranged from $96.7 \%$ for vancomycin to $28.8 \%$ for penicillin G. Antibiotic susceptibilities of gram-negative bacteria were overall very high, with $>90 \%$ susceptibility among isolated culture samples. Final VA outcomes of 20/400 or better were reported in $62.5 \%$ of patients.

Conclusions: The study demonstrates that the most frequent clinical etiology of endophthalmitis was exogenous due to progression of corneal ulcer and postoperative infection after cataract extraction. The spectrum of pathogens causing endophthalmitis is composed of mainly Gram-positive organisms (particularly coagulasenegative Staphylococcus). VA was improved in the majority of patients after treatment for endophthalmitis.
\end{abstract}

Keywords: acute endophthalmitis, microbial spectrum, antibiotic resistance

\section{Introduction}

$\mathbf{E}$ NDOPHTHALMITIS, A SERIOUS and vision-threatening ophthalmic emergency, is an intraocular inflammatory condition resulting from infection of the fluids and tissues of the eye. There are multiple types of endophthalmitis, broadly separated into exogenous and endogenous etiologies. Exogenous endophthalmitis occurs from direct inoculation of infectious organisms from intraocular procedures, trauma, or foreign bodies. ${ }^{1,2}$ Acute postoperative endophthalmitis is the most common form of endophthalmitis, often resulting as a complication of cataract surgery. ${ }^{2,3}$ Endogenous endophthalmitis, which accounts for $<10 \%$ of endophthalmitis cases, occurs when infectious organisms spread hematogenously to enter the intraocular space and is most commonly associated with underlying systemic and medical conditions, such as diabetes mellitus and immunosuppression. ${ }^{3-5}$

The incidence of endophthalmitis has been increasing in recent decades according to various studies, including a 2003 meta-analysis and a 2005 population-based review of Medicare claims data. ${ }^{6,7}$ The rise in incidence rate of postoperative endophthalmitis following cataract surgeries has been attributed by some investigators to the introduction of sutureless clear corneal incisions as a widely-performed surgical technique. ${ }^{8}$

\footnotetext{
${ }^{1}$ Department of Ophthalmology and Visual Science, Yale School of Medicine, New Haven, Connecticut, USA.

${ }^{2}$ Retina Department, New England Eye Center, Tufts Medical Center, Boston, Massachusetts, USA.

${ }^{3}$ Retina Department, Ophthalmic Consultants of Boston, Boston, Massachusetts, USA.
} 
Bacteria and fungi are the most common causative organisms of endophthalmitis, although the specific pathogens vary by etiology. For example, coagulase-negative Staphylococcus is the most common cause of postoperative endophthalmitis, as well as postintravitreal injection endophthalmitis (followed by viridans Streptococcus in postinjection patients), Bacillus cereus is common in post-traumatic endophthalmitis, and Candida species are common in endogenous fungal endophthalmitis., ${ }^{2,9}$

Suspected endophthalmitis requires immediate treatment with intraocular injection of antibiotics with or without pars plana vitrectomy to prevent worsening inflammation and blindness. ${ }^{10}$ An empiric broad-spectrum antibiotic regimentypically an intravitreal injection of vancomycin and ceftazidime-is often used before laboratory culture results are available. However, the increasingly widespread use of antibiotics has facilitated the emergence of reduced bacterial susceptibility and resistance to first-line antibiotic therapies. ${ }^{9,11,12}$ Thus, periodic assessment of dynamic trends in the microbial spectrum of organisms causing endophthalmitis and their antibiotic susceptibility and resistance is of utmost importance in constructing a maximally-effective empiric treatment regimen.

This study reports and analyzes the current microbial spectrum of organisms causing endophthalmitis and their antibiotic susceptibility and resistance among cultured organisms between 2011 and 2016. We compared our analysis with data previously published by our group, which reviewed cases between 1988 and 2008. In addition, we investigated the clinical epidemiology of acute endophthalmitis, evaluating clinical etiologies and predisposing risk factors, as well as patient outcomes of post-treatment visual acuity (VA), as such analyses have not been previously performed in this region.

\section{Methods}

This study is a retrospective analysis based on a medical chart review of patients with International Classification of Diseases (ICD)-9 and ICD-10 codes for endophthalmitis over a 5-year period (2011-2016) at a tertiary referral center. The use of these data was approved by Institutional Review Board at the Yale School of Medicine, and this study adhered to the tenets of the Declaration of Helsinki. We included all patients admitted at Yale-New Haven Hospital between January of 2011 and January of 2016 who were assigned a billing code of endophthalmitis (ICD-9 codes 360.0-360.1; ICD-10 codes H44.0-H44.1).

The clinical records for these patients were reviewed to evaluate clinical etiologies, microbial spectrum, antibiotic susceptibilities and resistance, and visual outcomes of acute endophthalmitis. Collected data included demographic information, microbiological analysis, and final corrected Snellen VA at last follow-up.

Microbiological analysis, including antibiotic susceptibility and resistance testing, was performed by the Clinical Microbiology Laboratory at Yale-New Haven Hospital. Specifically, antibiotic susceptibility testing was performed by automated broth microdilution on the Vitek2 (Biomerieux), with some additional testing done by the gradient diffusion by Etest (Biomerieux) or Kirby-Bauer disk diffusion. All interpretations of antibiotic susceptibility results were made according to current Clinical and Laboratory
Standards Institute M100 or M45 Performance Standards for Antimicrobial Susceptibility Testing. The Vitek2 was developed in 1998 but the Vitek system has existed since 1980. The Clinical Microbiology Laboratory at Yale-New Haven Hospital has consistently used the Vitek system and Kirby-Bauer disk diffusion for antibiotic susceptibility testing on vitreous samples. All data collection and analysis were performed using Microsoft Excel 2011 and SPSS version 16.0.1 (SPSS, Chicago, IL).

\section{Results}

A total of 94 patients were identified with culture-proven endophthalmitis over the 5-year period between 2011 and 2016. The microbial spectrum of causative organisms was dominated by coagulase-negative Staphylococcus (30.9\% of total samples), followed by Staphylococcus aureus (23.4\%), Pseudomonas aeruginosa (10.6\%), and Streptococcus pneumoniae $(7.4 \%)$. Gram-positive bacteria, gram-negative bacteria, and fungi comprised $74.4 \%, 18.1 \%$, and $7.4 \%$ of all isolated species, respectively (Table 1 ). The most frequent fungal isolates were Candida species (4.3\%) and Aspergillus fumigatus (2.1\%).

Antibiotic susceptibilities of gram-positive bacteria were the following: vancomycin, $96.7 \%$; cefazolin, $79.3 \%$; clindamycin, 69.0\%; doxycycline, 96.6\%; erythromycin 55.9\%; gentamicin, 96.6\%; oxacillin 69.0\%; penicillin G, 28.8\%; and trimethoprim/sulfamethoxazole $84.4 \%$ (Fig. 1A). Antibiotic susceptibilities of gram-negative bacteria were overall very high, with all antibiotics except for trimethoprim/ sulfamethoxazole achieving $90 \%$ or greater susceptibility among isolated culture samples: amikacin, $100 \%$; ceftazidime, 92.3\%; ciprofloxacin, 91.7\%; gentamicin, 90.9\%; meropenem, 100\%; piperacillin/tazobactam, $90.0 \%$; tobramycin, 100\%; trimethoprim/sulfamethoxazole, $80 \%$;

Table 1. Microbial Spectrum of Causative Species

\begin{tabular}{|c|c|}
\hline Causative organism & $\begin{array}{c}\text { All isolates } \\
n(\%)\end{array}$ \\
\hline Gram-positive organisms & $70(74.5)$ \\
\hline Staphylococcus, coagulase-negative & $29(30.9)$ \\
\hline Staphylococcus aureus & $22(23.4)$ \\
\hline Streptococcus pneumoniae & $7(7.4)$ \\
\hline Streptococcus viridans & $4(4.3)$ \\
\hline Bacillus species & $3(3.2)$ \\
\hline Corynebacterium species & $2(2.1)$ \\
\hline Propiobacterium acnes & $1(1.1)$ \\
\hline $\begin{array}{l}\text { Streptococcus equisimilis } \\
\text { (beta-hemolytic Group G) }\end{array}$ & $1(1.1)$ \\
\hline Streptomyces & $1(1.1)$ \\
\hline Gram-negative organisms & $17(18.1)$ \\
\hline Pseudomonas sp. & $10(10.6)$ \\
\hline Moraxella nonliquefaciens & $4(4.3)$ \\
\hline Haemophilus influenzae & $1(1.1)$ \\
\hline Klebsiella variicola & $1(1.1)$ \\
\hline Pasteurella canis & $1(1.1)$ \\
\hline Fungi & $7(7.4)$ \\
\hline $\begin{array}{l}\text { Candida species }(\text { C. albicans, } \\
\text { C. parapsilosis, C. tropicalis })\end{array}$ & $4(4.3)$ \\
\hline Aspergillus fumigatus & $2(1)$ \\
\hline Scedosporium apiospermum & $1(1.1)$ \\
\hline Total & $94(100)$ \\
\hline
\end{tabular}


FIG. 1. (A) Antibiotic susceptibilities of isolated Gram-positive bacteria for culture-proven endophthalmitis. (B) Antibiotic susceptibilities of isolated Gram-negative bacteria for culture-proven endophthalmitis.
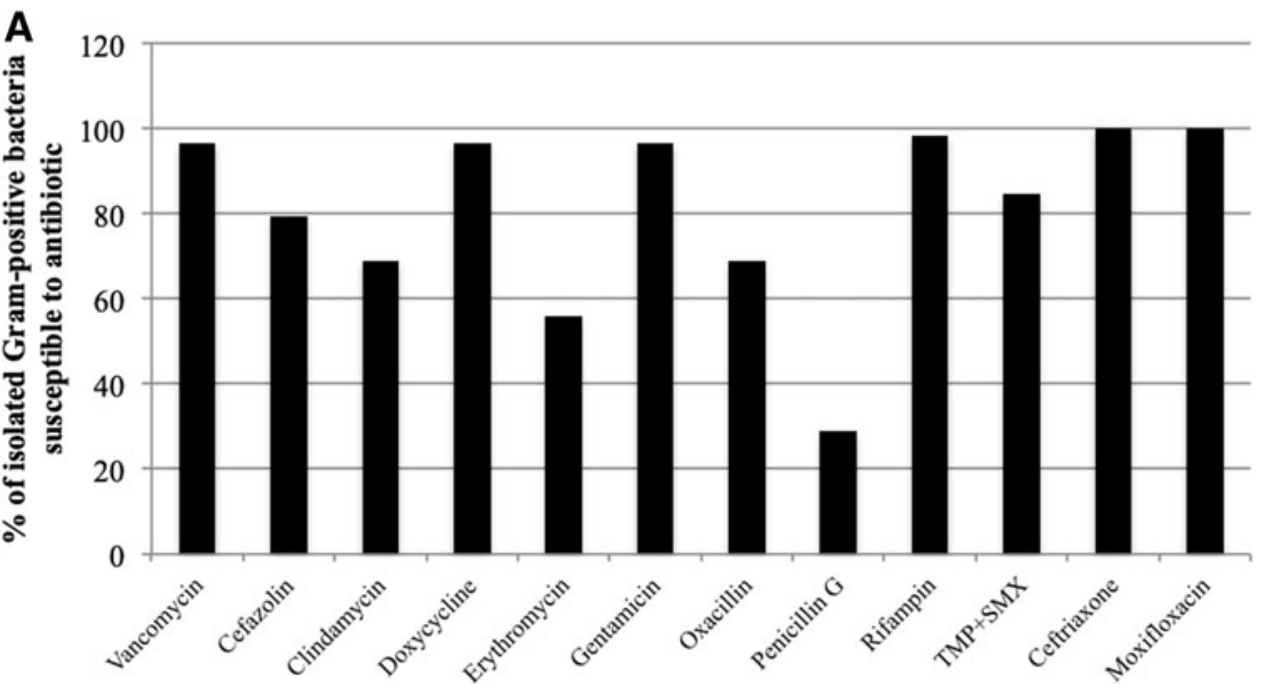

B

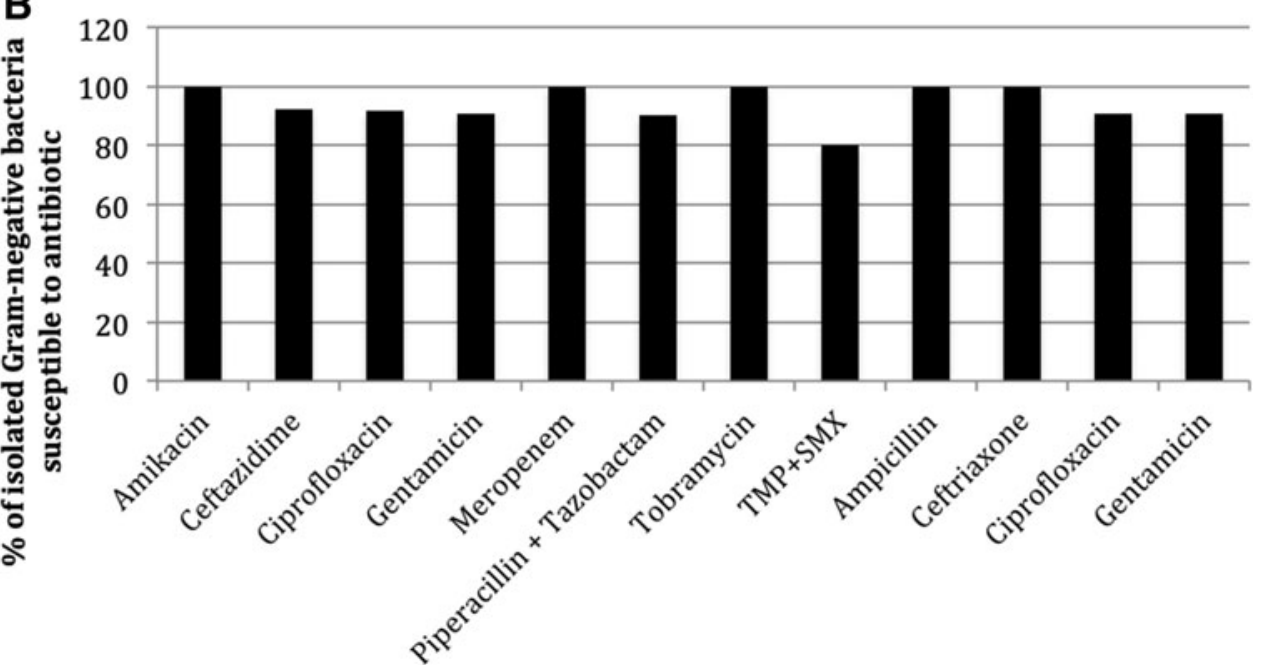

ampicillin, 100\%; ceftriaxone, 100\%; ciprofloxacin, 90.9\%; and gentamicin, $90.9 \%$. Among the fungal isolates tested, $100 \%$ were susceptible to voriconazole (Fig. 1B).

The etiologies of endophthalmitis were exogenous in $68.8 \%$ of cases and endogenous in $31.2 \%$ of cases (Fig. 2). Among cases of exogenous endophthalmitis, the most common inciting factors were progression of corneal ulcer and postoperative infection after cataract extraction $(31.8 \%$ and $25 \%$ of all exogenous cases, respectively). Other inciting factors included glaucoma drainage devices (11.4\%), other surgical procedures $(9.1 \%)$, intravitreal injection $(6.8 \%)$, pars plana vitrectomy $(4.5 \%)$, and trauma to the eye $(4.5 \%)$. Among cases of endogenous endophthalmitis, bacteremia was the causative factor of the vast majority of endogenous cases ( $95 \%$ of all endogenous cases), with one case due to invasive disseminated aspergillosis $(5 \%)$.

Final post-treatment VA outcomes of $20 / 400$ or better were reported in $62.5 \%$ of patients; counting fingers, hand motion, or light perception was reported in $10.0 \%$ of patients; and no light perception was reported in $27.5 \%$ of patients for whom VA data were available (Table 2). Among patients who had both initial and final VAs recorded, $79.2 \%$ of patients had improved VA, while $20.8 \%$ of patients had same or worsened VA at the last follow-up visit. Stratifying outcomes based on etiology of endoph- thalmitis, VA of $20 / 400$ or better was achieved in $62.5 \%$ of patients who developed endophthalmitis from progression of a corneal ulcer, $81.3 \%$ of patients with postoperative infection after cataract extraction, $50.0 \%$ of patients with postoperative infection after traditional glaucoma surgery, $33.3 \%$ of patients with infection after intravitreal injection, and $66.7 \%$ of patients who developed endophthalmitis secondary to bacteremia.

Cases of endophthalmitis in this current study were dominated by Gram-positive organisms, particularly coagulasenegative Staphylococcus. Gram-positive organisms cultured from isolates were highly susceptible to vancomycin $(96.7 \%)$, but exhibited a range of susceptibilities for other antibiotics (as low as $28.8 \%$ for penicillin G). Gram-negative organisms achieved very high (>90\%) antibiotic susceptibility overall.

\section{Discussion}

Endophthalmitis is a serious and vision-threatening ophthalmic emergency that necessitates a low index of suspicion to initiate immediate treatment with intravitreal antibiotics to prevent worsening inflammation and blindness. ${ }^{13}$ An empiric antibiotic regimen of intravitreal vancomycin and ceftazidime is commonly administered, while ophthalmologists await final laboratory culture results and susceptibilities to 


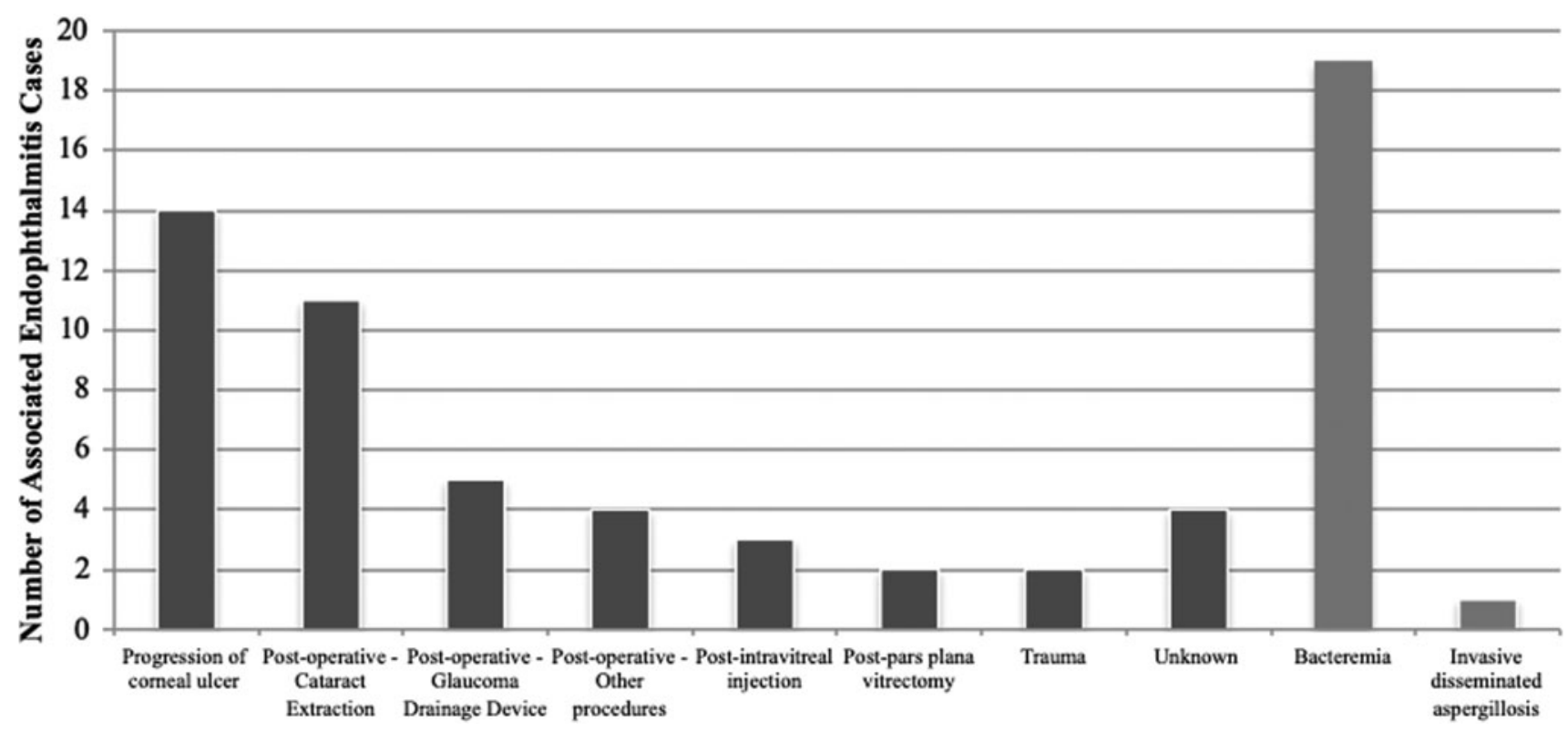

FIG. 2. Clinical etiologies of endophthalmitis: exogenous (black) and endogenous (grey) etiologies.

determine a more targeted regimen. Epidemiologic surveillance from this study adds to a growing body of evidence that the increasingly widespread use of antibiotics has facilitated the emergence of reduced bacterial susceptibility and resistance to several commonly used drugs.

Microbiological data obtained from the same tertiary referral center between 1988 and 2008 identified 160 isolated culture samples of endophthalmitis, with the microbiological spectrum comprising $80.6 \%$ Gram-positive organisms $(n=129), 12.5 \%$ Gram-negative organisms $(n=20)$, and $6.9 \%$ fungal organisms $(n=11){ }^{14}$ The most prevalent causative organisms were coagulase-negative Staphylococcus (37.5\%), viridans Streptococcus (11.3\%), and Streptococcus pneumoniae (6.9\%). Other Gram-positive organisms included Propionibacterium acnes (5.6\%), other Streptococcus species (4.4\%), Staphylococcus aureus (4.4\%), and E. faecalis (3.8\%). The most prevalent Gram-negative organisms were Klebsiella species (3.1\%), Moraxella species (3.1\%), and Haemophilus species (2.5\%).

Antibiotic susceptibility and resistance testing from the prior study period (1988-2008) showed that vancomycin

Table 2. Visual Acuity Outcomes OF ENDOPHTHALMITIS

\begin{tabular}{lc}
\hline Final VA & $\begin{array}{c}\text { Patients } \\
n(\%)\end{array}$ \\
\hline 20/400 or better & $30(62.5)$ \\
CF, HM, or LP & $5(10.0)$ \\
NLP & $13(27.5)$ \\
Total & $48(100)$ \\
Change in VA & \\
Improvement in VA & $38(79.2)$ \\
No change in VA & $6(12.5)$ \\
$\quad$ Worsening VA & $4(8.3)$ \\
Total & $48(100)$ \\
\hline
\end{tabular}

CF, counting fingers; HM, hand movements; LP, light perception; NLP, no light perception; VA, visual acuity. was effective against all coagulase-negative Staphylococcus, Streptococcus, and E. faecalis isolates that were tested. All Staphylococcus aureus isolates were sensitive to oxacillin. The researchers also noted increasing resistance over time of coagulase-negative Staphylococcus, with $100 \%$ of organisms exhibiting resistance to at least one of the antibiotics tested during 2002-2008, compared to $73.1 \%$ of organisms during 1995-2001. In particular, coagulase-negative Staphylococcus showed increasing resistance to penicillin and tetracycline and decreasing resistance to chloramphenicol and gentamicin over the course of the studied time period.

Comparing data obtained from the current study (20112016) to data recorded from previous microbiological data (1988-2008) at the same tertiary referral center, both the current microbial spectrum of culture-proven endophthalmitis, as well as antibiotic susceptibilities, have shifted, among other features, toward a higher percentage of Gramnegative bacteria (Table 2).

While in the earlier investigation, $P$. aeruginosa was an insignificant cause of endophthalmitis, data show that it comprised $10.6 \%$ of the microbial spectrum of causative organisms between 2011 and 2016. In addition, while there was previously no evidence of antibiotic resistance to vancomycin among isolates of coagulase-negative Staphylococcus, Streptococcus, and E. faecalis, and no evidence of resistance to oxacillin among isolates of Staphylococcus aureus, the current data indicate that at least $3.3 \%$ of isolates exhibited resistance to vancomycin, and $31 \%$ of Staphylococcus aureus isolates exhibited resistance to oxacillin.

Comparing current epidemiological data to data from the previous time period also reveals a continuing increase in resistance over time of coagulase-negative Staphylococcus, with $100 \%$ of isolates exhibiting resistance to at least 2 antibiotics tested and $77 \%$ of isolates exhibiting resistance to at least 3 antibiotics tested in the current study period. In particular, coagulase-negative Staphylococcus has showed emerging resistance to clindamycin, erythromycin, and penicillin G. In addition, patients from the current study period 
Table 3. Comparison of Current Study (2011-2016) with Previous Study (1988-2008)

\begin{tabular}{lcccc}
\hline & $\begin{array}{c}\text { Current study } \\
(2011-2016) \\
n(\%)\end{array}$ & $\begin{array}{c}\text { Chen and Adelman } \\
(2002-2008) \\
n(\%)\end{array}$ & $\begin{array}{c}\text { Chen and Adelman } \\
(1995-2001) \\
n(\%)\end{array}$ & $\begin{array}{c}\text { Chen and Adelman }^{14} \\
(1988-1994) \\
n(\%)\end{array}$ \\
\hline All isolates & 94 & 42 & 71 & 47 \\
Gram-positive isolates & $70(74.5)$ & $38(90.5)$ & $50(70.4)$ & $41(87.2)$ \\
Gram-negative isolates & $17(18.1)$ & $2(4.8)$ & $14(19.7)$ & $4(8.5)$ \\
Fungal isolates & $4(7.4)$ & $2(4.8)$ & $7(9.9)$ & $2(4.3)$ \\
$P$ value $^{\text {a }}$ & - & 0.204 & 0.566 & 0.467 \\
\hline
\end{tabular}

${ }^{\text {a }} P$ value calculated using 2 -sided exact Pearson chi-squared between current study and each time period in the previous study.

exhibited new resistance to ceftazidime, ciprofloxacin (a second-generation fluoroquinolone), and moxifloxacin (a fourth-generation fluoroquinolone).

Selection of empiric antibiotics for usage in immediate circumstances for acute endophthalmitis should be periodically influenced by information regarding the continuously evolving microbial spectrum and antibiotic susceptibilities. Current antibiotic protocols at the majority of major hospitals commonly begin with an empiric regimen of intravitreal vancomycin for Gram-positive coverage along with intravitreal ceftazidime for Gram-negative coverage, with amikacin used for individuals with penicillin hypersensitivity.

Data from the current study showed a shift in the prevalence of Gram-negative causative organisms, especially $P$. aeruginosa (which often requires the addition of a topical fluoroquinolone), from Gram-negative isolates comprising $4.8 \%$ of all causative organisms in $2002-2008$ to $18.1 \%$ in the current study period. However, this increase was not statistically significant and may reflect variability in levels of causative organisms, as data from an even earlier time period (1995-2001) found Gram-negative isolates to comprise $19.7 \%$ of all causative organisms (Table 3 ).

Cases of endophthalmitis caused by $P$. aeruginosa were found to primarily originate from progression of corneal ulcer, with less common etiologies being postoperative infection after cataract surgery and glaucoma surgery. $P$. aeruginosa, in particular, is known to produce a robust biofilm, a polymer matrix composed of polysaccharide, protein, and DNA that are self-produced by the bacterium and exhibit high resistance to antibiotics. ${ }^{15}$ Coagulase-negative Staphylococcus is also notorious for their biofilm forming ability. The trend of increasing antibiotic resistance, especially among such biofilm-producing, Gram-negative causative bacteria, may be reflective of the evolution of molecular mechanisms by biofilms to become resistant to antibiotics. Appropriate precautions for proper preoperative and preprocedure disinfection to destroy biofilm-producing bacteria are paramount, and further studies investigating the prevention of biofilm formation will continue to inform the shifting landscape of bacterial virulence and antibiotic resistance. ${ }^{16}$

Overall, this study demonstrates that the most frequent clinical etiology of endophthalmitis was exogenous due to progression of corneal ulcer and postoperative infection after cataract extraction, although cases of endophthalmitis associated with these pathologies and procedures are rare relative to the large number of overall cases that do not result in endophthalmitis. A limitation of this study was that all cases of endophthalmitis included in the analysis were from patients at a single tertiary referral center, thereby providing only a constituent epidemiologic survey of endophthalmitis in a particular region of the United States. Similar studies should be performed at other treatment centers to observe an overall trend in the microbial spectrum and antibiotic susceptibilities in cases of acute endophthalmitis. Furthermore, future studies examining the trends of endophthalmitis according to causative factors may aid understanding in which pathologies or procedures pose the greatest risk of endophthalmitis over time and whether interventions may be implemented to directly mitigate specific factors.

The spectrum of pathogens causing endophthalmitis is composed of mainly Gram-positive organisms (particularly coagulase-negative Staphylococcus), although epidemiologic analysis of the trends in the microbiologic spectrum and antibiotic susceptibilities demonstrates higher prevalence of Gram-negative causative organisms in cases of exogenous endophthalmitis, as well as increasing resistance to commonly used antibiotics. Thus, on a patient-provider level, ophthalmologists must conduct close follow-up of patients with suspected endophthalmitis after the initial empiric antibiotic regimen, with heightened awareness of changing patterns in causative organisms and antibiotic susceptibilities.

\section{Disclaimer}

This submission has not been published anywhere previously and is not simultaneously being considered for any other publication.

\section{Author Disclosure Statement}

No competing financial interests exist.

\section{Funding Information}

This research was funded by the Leir Foundation, New York, NY and Yale University School of Medicine Office of Student Research, New Haven, CT.

\section{References}

1. Lemley, C.A., and Han, D.P. Endophthalmitis: a review of current evaluation and management. Retina. 27:662-680, 2007.

2. Durand, M.L. Endophthalmitis. Clin. Microbiol. Infect. 19: 227-234, 2013.

3. Kernt, M., and Kampik, A. Endophthalmitis-pathogenesis, clinical presentation, management, and perspectives. Clin. Ophthalmol. 4:121-135, 2010.

4. Jabbarvand, M., Hashemian, H., Khodaparast, M., et al. Endophthalmitis occurring after cataract surgery: outcomes of more than 480000 cataract surgeries, epidemiologic features, and risk factors. Ophthalmology. 123:295-301, 2016. 
5. Connell, P.P., O’Neill, E.C., Fabinyi, D., et al. Endogenous endophthalmitis: 10-year experience at a tertiary referral centre. Eye (Lond). 25:66-72, 2011.

6. Taban, M., Behrens, A., Newcomb, R.L., et al. Acute endophthalmitis following cataract surgery a systematic review of the literature. Arch. Ophthalmol. 123:613-620, 2005.

7. Abelson, M.B., Lane, K., and Slocum, C. The secrets of ocular microbiomes. Rev. Ophthalmol. 22:44-46, 2015.

8. Al Mahmood, A.M., Al-Swailem, S.A., and Behrens, A. Clear corneal incision in cataract surgery. Middle East Afr. J. Ophthalmol. 21:25-31, 2014.

9. Relhan, N., Albini, T.A., Pathengay, A., et al. Endophthalmitis caused by Gram-positive organisms with reduced vancomycin susceptibility: literature review and options for treatment. Br. J. Ophthalmol. 100:446-452, 2016.

10. Bradley, J.M., Vranka, J., Colvis, C.M., et al. Effect of matrix metalloproteinases activity on outflow in perfused human organ culture. Invest. Ophthalmol. Vis. Sci. 39: 2649-2658, 1998.

11. Bains, H.S., Weinberg, D.V., Feder, R.S., and Noskin, G.A. Postoperative vancomycin-resistant Enterococcus faecium endophthalmitis. Arch. Ophthalmol. 125:12921293, 2007.

12. Chang, D.F., Braga-Mele, R., Henderson, B.A., et al. Antibiotic prophylaxis of postoperative endophthalmitis after cataract surgery: results of the 2014 ASCRS member survey. J. Cataract. Refract. Surg. 41:1300-1305, 2015.
13. Das, T., Sharma, S., and Hyderabad Endophthalmitis Research Group. Current management strategies of acute post-operative endophthalmitis. Semin. Ophthalmol. 18: 109-115, 2003.

14. Chen, X., and Adelman, R.A. Microbial spectrum and resistance patterns in endophthalmitis: a 21-year (1988-2008) review in northeast United States. J. Ocul. Pharmacol. Ther. 28:329-334, 2012.

15. Høiby, N., Bjarnsholt, T., Givskov, M., Molin, S., and Ciofu, O. Antibiotic resistance of bacterial biofilms. Int. J. Antimicrob. Agents. 35:322-322, 2010.

16. Kivanc, S.A., Kivanc, M., and Bayramlar, H. Microbiology of corneal wounds after cataract surgery: biofilm formation and antibiotic resistance patterns. J. Wound Care. 12:1419, 2016.

Received: July 12, 2019

Accepted: February 12, 2020

Address correspondence to:

Dr. Ron A. Adelman

Department of Ophthalmology and Visual Science

Yale School of Medicine

40 Temple Street

New Haven, CT 06510

USA

E-mail: ron.adelman@yale.edu 